\title{
PLANE FROBENIUS SANDWICHES ${ }^{1}$
}

\section{R. GANONG}

\begin{abstract}
Suppose the total Frobenius of an affine plane factors through a plane. Then the factorization is just the composite of relative Frobeniuses with respect to suitable coordinate axes.
\end{abstract}

THEOREM. Suppose $k$ is an algebraically closed field of characteristic $p>0$, and $R, A$ are polynomial (respectively power series) rings over $k$ in two variables, with $R^{p} \underset{\neq}{\subset} \underset{\neq}{\subset}$. Then there exist $x, y \in R$ such that

$$
R=k[x, y] \text { and } A=k\left[x, y^{p}\right]
$$

$$
\text { (respectively } \left.R=k[[x, y]] \text { and } A=k\left[\left[x, y^{p}\right]\right]\right) \text {. }
$$

The key step in the proof of the polynomial case of the Theorem rests on recent work of Peter Russell [7, Theorem 5.3] on approximate roots of polynomials, work which may be regarded as extending earlier results of Abhyankar and Moh [1, 2]. The proof of the Theorem for power series is comparatively trivial.

Proof of The Theorem. If $x \in R$ and there exists $y \in R$ such that $R=k[x, y]$ (resp. $R=k[[x, y]]$ ), we say $x$ is a variable. Clearly we need only find a variable in A.

1. The polynomial case. Let $A=k[u, v]$.

(i) Suppose $u$ or $v$ is reducible in $R$, say $u$ is reducible. Let $a \in R$ be an irreducible factor of $u$ and write $u=a^{i} b, a \nmid b$. Then $u^{p}=\left(a^{p}\right)^{i} b^{p}$, so $b^{p}=c u^{j}, c \in k^{*}$, $0 \leqslant j<p$. Hence $b^{p-j}=c a^{i j}$, so $b \in k^{*}$ and we must have $i=p$. Take $x=v$, $y=a$.

(ii) Suppose $u, v \in R$ are irreducible. One sees easily then that $u, v \in R$ are curves with one place at infinity. (For the definition see $[1,11.4]$ or $[3,1.1]$.) Also, the intersection index of $u$ and $v$ on $\operatorname{Spec} R$ is $p$. (For, if the intersection were 1, we would have $R=A$. For two different proofs of this fact, see [3, 1.17] and [7, 6.3].)

Choose $x^{\prime}, y^{\prime} \in R$ such that $R=k\left[x^{\prime}, y^{\prime}\right]$ and embed $\operatorname{Spec} R$ in $\mathbf{P}^{2}$ in the usual way. $\left(x^{\prime}=X / Z, y^{\prime}=Y / Z\right.$, where $X, Y, Z$ are homogeneous coordinates.) Let $C, D$

Received by the editors October 2, 1979 and, in revised form, November 6, 1980.

AMS (MOS) subject classifications (1970). Primary 13F20, 13J05; Secondary 14E35, 14H45.

${ }^{1}$ Research supported in part by a joint grant, from the Asia Society, Inc., NSF (Grant No. INT-7705038), ICA (Grant Authorization No. 1069-840393), and the University Grants Commission (Government of India), and awarded by the Indo-American Fellowship Program for work at Bhaskaracharya Pratishthana, Pune, India.

I thank Mrs. Lydia Gomes, of the Council for the International Exchange of Scholars, and Messrs. Mehendiratta, Suri, and Bhandare, of the American Institute for Indian Studies, for their helpfulness and hospitality, and Hilde Schroeder, for help in the preparation of the manuscript. 
be the projective curves defined by $u, v$ respectively, and let $\operatorname{deg} u=c_{1}, \operatorname{deg} v=c_{1}^{\prime}$. We have $c_{1} c_{1}^{\prime} \geqslant p$.

If $c_{1} c_{1}^{\prime}=p$, then $u$ or $v$ is linear in $x^{\prime}, y^{\prime}$, hence is a variable. Suppose that $c_{1} c_{1}^{\prime}>p$ and that the Theorem holds whenever $A=k\left[u^{\prime}, v^{\prime}\right]$ with $\operatorname{deg} u^{\prime} \operatorname{deg} v^{\prime}<c_{1} c_{1}^{\prime}$. Performing a linear change of coordinates, we may choose new $x^{\prime}, y^{\prime}$ such that

$$
u=y^{\prime c_{1}}+\left(\text { terms of degree }<c_{1}\right),
$$

and hence (multiplying $v$ by a nonzero constant)

$$
v=y^{\prime c_{1}^{\prime}}+\left(\text { terms of degree }<c_{1}^{\prime}\right) \text {. }
$$

We put $x^{\prime \prime}=1 / x^{\prime}, y^{\prime \prime}=y^{\prime} / x^{\prime}, u^{\prime \prime}=u / x^{\prime c_{1}}, v^{\prime \prime}=v / x^{\prime c^{\prime}} . u^{\prime \prime}, v^{\prime \prime} \in k\left[\left[x^{\prime \prime}, y^{\prime \prime}\right]\right]$ are irreducible (and are local equations for $C, D$ at $X=1, Y=Z=0$ ).

We interrupt the proof at this juncture with a review of Hamburger-Noether $(\mathrm{HN})$ expansion. Since Noether's Palermo paper of 1890, a vast amount has been published about HN and about the less generally applicable, but much older, NewtonPuiseux expansion. We refer only to [7] for what is needed here; in the writer's opinion it represents the latest word on the subject, both in its content and in the particular way in which it proceeds from the basics to some of the deepest results in the theory of plane, and algebroid plane, curves. The reader is directed to the references given in [7] and to the introduction to that paper, where among other things is indicated the rôle played by the work of Moh and of Abhyankar, over the past fifteen years or so, in the revival of interest in the subject.

(a) Let $S=k[[t]]$ be the ring of power series in $t$ over $k$. Given nonunits $x, y \in S$, with $x \neq 0$, define the (algebroid) Hamburger-Noether tableau

$$
\mathrm{HN}(x, y)=\left[\begin{array}{c}
p_{i} \\
c_{i} \\
\alpha_{i}
\end{array}\right]_{1 \leqslant i<\infty}
$$

as follows:

If $y=0$, put $c_{i}=$ ord $x<\infty, p_{i}=$ ord $y=\infty, \alpha_{i}=0$ for $i \geqslant 1$ (ord means $t$-order).

Suppose $y \neq 0$. Put $p_{1}=$ ord $y, c_{1}=$ ord $x$. There exist unique (nonzero) elements $z_{2}, \ldots, z_{r+1} \in S$ such that (with $\left.z_{0}=y, z_{1}=x\right) z_{i-1}=z_{i}^{s_{i}} z_{i+1}, 1 \leqslant i \leqslant r$, with $s_{i}$ nonnegative integers, $0<$ ord $z_{i}<$ ord $z_{i-1}, 2 \leqslant i \leqslant r$, and ord $z_{r+1}=0$.

$$
\text { ord } z_{i-1}=s_{i} \text { ord } z_{i}+\text { ord } z_{i+1}, \quad 1 \leqslant i \leqslant r,
$$

is the Euclidean algorithm on $(\operatorname{ord} y, \operatorname{ord} x)$; hence ord $z_{r}=\operatorname{gcd}(\operatorname{ord} y$, ord $x)$. There is a unique $\alpha \in k^{*}$ such that

$$
\operatorname{ord}\left(z_{r-1}-\alpha z_{r}^{s_{r}}\right)>\text { ord } z_{r-1} \text {. }
$$

Put $\alpha_{1}=\alpha, x_{2}=z_{r}, y_{2}=\left(z_{r-1}-\alpha z_{r}^{s_{r}}\right) / z_{r-1}, p_{2}=$ ord $y_{2}, c_{2}=$ ord $x_{2}$, etc. (Cf. [7, §2].) Note again that $c_{2}$ divides $p_{1}$ and $c_{1}$.

(b) Let $f \in T=k[[x, y]]$ be an irreducible power series in two variables. Then we have an isomorphism (integral closure of $T / f T) \stackrel{\simeq}{\rightarrow} S$ carrying $(x, y)$ to a "primitive branch representation" $(x(t), y(t))$ for $f$. We put $\mathrm{HN}(x, y ; f)=\mathrm{HN}(x(t), y(t))[7$, $\S 3]$. We remark that the left-hand side may be defined without having recourse to a 
parametrization for $f$, using well-known facts about the Newton polygon of an irreducible power series in two variables, and its behavior under dilatations. (See, e.g., [3, 2.2-2.9].) Defining it either way, we have

(c) Given coprime irreducible $f, g \in T$, the intersection multiplicity

$$
i(f, g)=\operatorname{dim}_{k} T /(f T+g T)=\sum_{i<s} p_{i} c_{i}^{\prime}+\min \left\{p_{s} c_{s}^{\prime}, p_{s}^{\prime} c_{s}\right\},
$$

where $p_{i}, c_{i}, \alpha_{i}$ and $p_{i}^{\prime}, c_{i}^{\prime}, \alpha_{i}^{\prime}$ are the entries of $\mathrm{HN}(x, y ; f)$ and $\mathrm{HN}(x, y ; g)$ respectively, and the integer $s \geqslant 1$ is determined by $p_{i} c_{i}^{\prime}=p_{i}^{\prime} c_{i}$ and $\alpha_{i}=\alpha_{i}^{\prime}$ for $i<s$, $p_{s} c_{s}^{\prime} \neq p_{s}^{\prime} c_{s}$ or $\alpha_{s} \neq \alpha_{s}^{\prime}$. (See [7, 3.3]. Note that our $s$ is $s+1$, in [7].)

(d) Keep the notation of (c). For $i<s, c_{s}$ divides $p_{i}$ and $c_{i}$, and $c_{s}^{\prime}$ divides $p_{i}^{\prime}$ and $c_{i}^{\prime}$, by the last remark in (a). We also have $c_{i} c_{s}^{\prime}=c_{i}^{\prime} c_{s}[7,2.10]$.

Returning to the proof, we consider the intersection multipicity $i\left(u^{\prime \prime}, v^{\prime \prime}\right)$ of $C$ and $D$ at infinity. Let

$$
\mathrm{HN}\left(x^{\prime \prime}, y^{\prime \prime} ; u^{\prime \prime}\right)=\left[\begin{array}{c}
p_{i} \\
c_{i} \\
\alpha_{i}
\end{array}\right]_{1<i<\infty}, \quad \mathrm{HN}\left(x^{\prime \prime}, y^{\prime \prime} ; v^{\prime \prime}\right)=\left[\begin{array}{c}
p_{i}^{\prime} \\
c_{i}^{\prime} \\
\alpha_{i}^{\prime}
\end{array}\right]_{1 \leqslant i<\infty} .
$$

Since $u^{\prime \prime}, v^{\prime \prime}$ are coprime, there is a positive integer $s$ such that $p_{i} c_{i}^{\prime}=p_{i}^{\prime} c_{i}$ and $\alpha_{i}=\alpha_{i}^{\prime}$ for $i<s$, and $p_{s} c_{s}^{\prime} \neq p_{s}^{\prime} c_{s}$ or $\alpha_{s} \neq \alpha_{s}^{\prime}$. We may assume $p_{s} c_{s}^{\prime} \leqslant p_{s}^{\prime} c_{s}$. Then by (c) (cf. [9, p. 35]), we have $i\left(u^{\prime \prime}, v^{\prime \prime}\right)=\Sigma_{i \leqslant s} p_{i} c_{i}^{\prime}=c_{1} c_{1}^{\prime}-p$, the second equality holding by Bezout's theorem.

Suppose $s=1$. Then $p=c_{1} c_{1}^{\prime}-p_{1} c_{1}^{\prime}$, so $c_{1}^{\prime}$ divides $p$. If $c_{1}^{\prime}=1$ then $v$ is linear. If $c_{1}^{\prime}=p$ then $1=c_{1}-p_{1}=\operatorname{deg}_{x^{\prime}} u$. Since $u$ has one place at infinity, there exist $d \in k^{*}$ and $g \in k\left[y^{\prime}\right]$ such that $u=d x^{\prime}+g$ (see, e.g., [1, 11.12(i)]), and $u$ is a variable.

Suppose $s>1$. Since $c_{s}^{\prime}$ divides $c_{i}^{\prime}$ for $i<s$, we have that $c_{s}^{\prime}$ divides $p$. If $c_{s}^{\prime}=1$, then $c_{1}=c_{1} c_{s}^{\prime}=c_{1}^{\prime} c_{s}$ by (d). Let $u^{\prime}=u-v^{c_{s}}$. Then $A=k\left[u^{\prime}, v\right]$ and $\operatorname{deg} u^{\prime}<c_{1}$, so the Theorem holds by induction. So we may assume $c_{s}^{\prime}=p$. From $c_{1} c_{s}^{\prime}=c_{1}^{\prime} c_{s}$, we see that if $c_{s}=1$ or $p$ divides $c_{s}$, then $c_{1}$ divides $c_{1}^{\prime}$ or $c_{1}^{\prime}$ divides $c_{1}$, and we proceed as above. So we may assume $p \nmid c_{s}>1$. We have

$$
1=c_{1}\left(c_{1}^{\prime} / p\right)-\sum_{i<s} p_{i}\left(c_{i}^{\prime} / p\right)-p_{s}
$$

since $c_{s}$ divides $p_{i}$ for $i<s$ and $c_{s}$ divides $c_{1}$, we have $c_{s+1}=\operatorname{gcd}\left(p_{s}, c_{s}\right)=1$. Therefore

$$
c_{s} \text { is the "last characteristic multiplicity" of } u^{\prime \prime} \text { with respect to } x^{\prime \prime}, y^{\prime \prime} \text {. }
$$

As in $[7,2.8 .4,2.8 .5]$, we put $r_{h}=\Sigma_{i<s} p_{i} c_{i} / c_{s}$. Note that $r_{h}=\Sigma_{i \leqslant s} p_{i}\left(c_{i}^{\prime} / p\right)=$ $c_{1} c_{1}^{\prime} / p-1=c_{1}^{2} / c_{s}-1$. By [7, Theorem 5.3], we conclude that there exists a $y \in R$ with one place at infinity, of degree $c_{1} / c_{s}$, such that the projective curve defined by $y$ meets $C$ at infinity with intersection multiplicity $r_{h}$. Therefore the intersection of $u$ and $y$ on Spec $R$ is $c_{1}^{2} / c_{s}-r_{h}=1$. By [3, 1.17] or [7, 6.3], $R=k[u, y]$.

2. The power series case. Let $A=k[[u, v]]$. We claim $u$ or $v$ is a variable.

(i) Suppose $u$ or $v$ is reducible in $R$, say $u$ is reducible. Let $u=a^{i} b$ as in 1(i). Arguing as we did there, we find $b \in R^{*}$. If $p$ does not divide $i$, there exists $w \in R^{*}$ 
such that $w^{i}=b$. Then $u=(w a)^{i}$; hence $i$ must equal $p$, a contradiction. So $p$ divides $i$ and $b=u / a^{i} \in \mathrm{qt} A$ is integral over $A$; hence $b \in A^{*}$. Also $u / b=a^{i}$ implies $i=p$, so $R=k[[a, v]]$.

(ii) Suppose $u, v \in R$ are irreducible. As in 1 (ii), we must have $i(u, v)=p$. Let $R=k\left[\left[x^{\prime}, y^{\prime}\right]\right]$, and let

$$
\mathrm{HN}\left(x^{\prime}, y^{\prime} ; u\right)=\left[\begin{array}{c}
p_{i} \\
c_{i} \\
\alpha_{i}
\end{array}\right], \quad \mathrm{HN}\left(x^{\prime}, y^{\prime} ; v\right)=\left[\begin{array}{c}
p_{i}^{\prime} \\
c_{i}^{\prime} \\
\alpha_{i}^{\prime}
\end{array}\right] .
$$

Define $s$ as before, assume $p_{s} c_{s}^{\prime} \leqslant p_{s}^{\prime} c_{s}$, let $d(u, v)=p$ - ord $u$ ord $v$, and note that $d(u, v) \geqslant 0$. If $d(u, v)=0$ or $s=1$, then $u$ or $v$ is of order one. So suppose $d(u, v)>0, s>1$. We have $p=\Sigma_{i<s} p_{i} c_{i}^{\prime}+p_{s} c_{s}^{\prime}$, so $c_{s}^{\prime}$ divides $p$, and necessarily $c_{s}^{\prime}=1$. Since $p_{1}=p_{1}^{\prime} c_{s}$ and $c_{1}=c_{1}^{\prime} c_{s}$, ord $u=\min \left\{p_{1}, c_{1}\right\}=c_{s} \min \left\{p_{1}^{\prime}, c_{1}^{\prime}\right\}=$ $c_{s}$ ord $v$. Since $d(u, v)>0, u$ and $v$ have the same tangent. So there exists unique $c \in k^{*}$ such that $\operatorname{ord}\left(u-c v^{c_{s}}\right)>$ ord $u$. Put $u^{\prime}=u-c v^{c_{s}} . A=k\left[\left[u^{\prime}, v\right]\right]$ and $d\left(u^{\prime}, v\right)<d(u, v)$. By induction and the fact that ord $u^{\prime}>$ ord $u \geqslant$ ord $v, v$ is a variable.

Question. Suppose $n>1, q=p^{n}$, and let $R^{q} \subset A \subset R, K=$ qt $R, L=$ qt $A$, $[K: L]=q, R, A$ otherwise as in the Theorem. Under what conditions does $A$ contain a variable? It is not sufficient to require that $K / L$ and $L / K^{q}$ be simple extensions, as the following example shows:

Let $n=2, a>1, p \nmid a, u=x^{a}+y^{p}, v=x^{p}, A=k[u, v]$ (resp. $k[[u, v]]$ ). If $f \in A$ then $x$ divides both $f_{x}$ and $f_{y}$, so $A$ cannot contain a variable.

Conjecture. The Theorem undoubtedly remains valid if [ ] and [[ ]] are everywhere replaced by ( ) and (( )), and rings replaced by fields.

REMARKS. The exposition of Hamburger-Noether appears at the insistence of the referee.

The writer proved the power series version of the theorem over a year after the polynomial case, as an afterthought. I thank Peter Russell for having suggested to me originally the problem in the polynomial case. Again, over a year after proving the power series case, I learned that it had already been proven in a paper of Rudakov and Šafarevič [6, corollary to Theorem 4], using work of C. S. Seshadri [8, Proposition 6]. At the close of [6] it is remarked by the translators that P. Deligne has also proven the power series case-whether for two, or an arbitrary number of, variables is unclear from the remark; I have not seen Deligne's proof.

Finally, I would like to apologize here to Professor T. T. Moh, for having unintentionally neglected to cite his paper [4] as a reference in my paper [3]; the papers [5] and [4] of Moh both appear here. The reader may profitably compare the content and approach of [5] with those of [7].

\section{REFERENCES}

1. S. S. Abhyankar, Lectures on expansion techniques in algebraic geometry (Notes by B. Singh), Tata Lecture Notes 57, Tata Inst. of Fundamental Research, Bombay, 1977.

2. S. S. Abhyankar and T. T. Moh, Newton-Puiseux expansion and generalized Tschirnhausen transformation, J. Reine Angew. Math. 260 (1973), 47-83; 261 (1973), 29-54. 
3. R. Ganong, On plane curves with one place at infinity, J. Reine Angew. Math. $307 / 308$ (1979), 173-193.

4. T. T. Moh, On analytic irreducibility at $\infty$ of a pencil of curves, Proc. Amer. Math. Soc. 44 (1974), 22-24.

5. On characteristic pairs of algebroid plane curves for characteristic $p$, Academia Sinica 1 (1973), 75-91.

6. A. N. Rudakov and I. R. Šafarevič, Inseparable morphisms of algebraic surfaces, Math. USSR-Izv. 10 (1976), 1205-1237.

7. P. Russell, Hamburger-Noether expansions and approximate roots of polynomials, Manuscripta Math. 31 (1980), 25-95.

8. C. S. Seshadri, L'opération de Cartier. Applications, Séminaire C. Chevalley 1958/59, Exposé 6.

9. W. van der Kulk, On polynomial rings in two variables, Nieuw Arch. voor Wisk. (3) 1 (1953), 33-41.

Department of Mathematics, McGill University, Montreal, Quebec, Canada H3A 2K6 